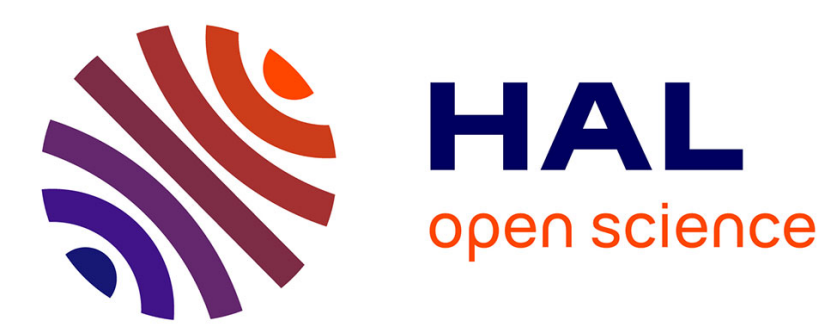

\title{
Dynamics of a tracer particle interacting with excitations of a Bose-Einstein condensate
}

\author{
Jonas Lampart, Peter Pickl
}

\section{To cite this version:}

Jonas Lampart, Peter Pickl. Dynamics of a tracer particle interacting with excitations of a BoseEinstein condensate. Annales Henri Poincaré, 2022, 23, pp.2855-2876. 10.1007/s00023-022-01153-5 . hal-03035282v2

\author{
HAL Id: hal-03035282 \\ https://hal.science/hal-03035282v2
}

Submitted on 9 Jul 2021

HAL is a multi-disciplinary open access archive for the deposit and dissemination of scientific research documents, whether they are published or not. The documents may come from teaching and research institutions in France or abroad, or from public or private research centers.
L'archive ouverte pluridisciplinaire HAL, est destinée au dépôt et à la diffusion de documents scientifiques de niveau recherche, publiés ou non, émanant des établissements d'enseignement et de recherche français ou étrangers, des laboratoires publics ou privés. 


\title{
Dynamics of a tracer particle interacting with excitations of a Bose-Einstein condensate
}

\author{
Jonas Lampart ${ }^{* \dagger}$, Peter Pickl ${ }^{* \ddagger}$
}

July 9,2021

\begin{abstract}
We consider the quantum dynamics of a large number $N$ of interacting bosons coupled a tracer particle, i.e. a particle of another kind, on a torus. We assume that in the initial state the bosons essentially form a homogeneous Bose-Einstein condensate, with some excitations. With an appropriate mean-field scaling of the interactions, we prove that the effective dynamics for $N \rightarrow \infty$ is generated by the Bogoliubov-Fröhlich Hamiltonian, which couples the tracer particle linearly to the excitation field.
\end{abstract}

\section{Introduction}

The reaction of a many-particle system to small perturbations - for example the introduction of a different particle - carries detailed information about its global properties. An interesting example is a Bose-Einstein condensate with an impurity particle. Apart from the obvious motivation of understanding this state of matter, Bose-Einstein condensates in the laboratory have the advantage that many of their parameters are controllable, which allows for comparison of theory and experiment over a large range paramter values. Systems of Bose-Einstein condensates with few impurities can now be realised using cold atoms and promise numerous applications [45]. Theoretical approches to their description are discussed in [21].

In this manuscript we will perform a mathematical analysis of the dynamics of such an impurity entering a Bose gas at zero temperature. In keeping with the convention of [13, 10], and since one motivation is to use the impurity to probe the condensate, we will call it "tracer" particle in what follows.

We will show that, for a certain choice of parameters and for a Bose-Einstein condensate of constant density, the tracer particle will effectively interact with the excitations in the gas,

\footnotetext{
*Ludwig-Maximilians-Universität, Mathematisches Institut, Theresienstr. 39, 80333 München, Germany.

${ }^{\dagger}$ CNRS \& Laboratoire Interdisciplinaire Carnot de Bourgogne (UMR 6303), 9. Av. A. Savary, 21078 Dijon Cedex. E-mail: lampart@math.cnrs.fr

¥Eberhard-Karls-Universität, Mathematisches Institut, Auf der Morgenstelle 10, 72076 Tübingen, Germany. E-Mail: p.pickl@uni-tuebingen.de
} 
which will be of finite number and are governed by the dynamics proposed by Bogoliubov in his seminal work [4]. Thus the system is - for a large number of bosons - in good approximation described by the Bogoliubov-Fröhlich Hamiltonian, given in (9) below. Our result is in agreement with recent findings by Mysliwy and Seiringer, who analyzed the spectral properties of the same system [30].

In order to get an interaction which is governed by the excitations in the condensate we have to assume that the condensate state has constant density - at least in the interaction region of the tracer particle. Otherwise, the effect coming from particles in the condensate state - which is at zero temperature the vast majority - would be dominant. Assuming constant density, this leading contribution of the interaction will also be a constant which simply leads to a global change in phase for the dynamics, and thus does not influence the motion of the tracer. To simplify the technicalities we assume that our system lives on a three dimensional torus of fixed length. However, we expect that this can be generalized. This generalization has to ensure, of course, that density stays constant in the range of the tracer particle for the full time scale of our observation, which amounts to considering gases of large volume.

The configuration space of one particle is the $d$-dimensional torus of side-length one, $T^{d}=\mathbb{R}^{d} / \mathbb{Z}^{d}$. The wave function of a system of $N$ bosons and one tracer particle in $T^{d}$ is then an element of

$$
\mathcal{H}_{N}=L^{2}\left(T^{d}\right) \otimes L^{2}\left(T^{d}\right)^{\otimes_{s} N},
$$

where $\otimes_{s}$ denotes the symmetric tensor product.

The dynamics of the system is described by the Schrödinger equation on $\mathcal{H}_{N}$

$$
\left\{\begin{aligned}
i \frac{d}{d t} \Psi(t) & =H_{N} \Psi(t) \\
\Psi(0) & =\Psi_{N}
\end{aligned}\right.
$$

with Hamiltonian

$$
H_{N}=-\frac{1}{2 m} \Delta_{x}-\sum_{j=1}^{N} \Delta_{y_{j}}+g_{B} \sum_{j \neq k=1}^{N} V\left(y_{j}-y_{k}\right)+g_{I} \sum_{j=1}^{N} W\left(x-y_{j}\right),
$$

where $x$ denotes the position of the tracer and $y_{1}, \ldots, y_{n}$ those of the bosons.

As an $N$-boson initial state $\Psi_{N}$ one can think of an $N$-fold product of the normalized constant on the torus times an $L^{2}$-function of $x \in T^{d}$ describing the initial state of the tracer. More generally, this could be modified by adding some "excitations", i.e. factors orthogonal to the constant function of $y \in T^{d}$, as long as their number stays finite as $N \rightarrow \infty$ (see (20) below for the precise condition).

The coupling constants $g_{B}$ of the boson interaction and $g_{I}$ of the interaction with the impurity will depend on the number $N$ of bosons. The precise form of the dependence has to be chosen appropriately to get the desired effect, i.e. a system where the interaction with excitations is of order one. 


\subsection{Mean-field equations and corrections}

The effective description of many-body systems is an old and yet vivid research area of mathematical physics. Hepp [22] and later Ginibre and Velo [14, 15] as well as Spohn [44] proved that the dynamics of a many-body Bose gas at zero temperature is, in the weak coupling limit, described by the Hartree equation in the sense that the one-particle marginal density of the $N$-body wave function converges to a pure state given by the solution of the Hartree equation as $N$ tends to infinity. The latter can be derived by replacing the interaction of the many-body system by the respective mean-field, effectively turning the $\mathrm{N}$ body description into a one-body dynamics. Subsequently, many improvements have been found, for example generalizations to a larger class of interactions, and different notions of convergence (see [25] for a review). The dilute (Gross-Pitaevskii) limit, where interactions are of order one but rare, is a variation of this theme that has also been studied extensively (see [1]).

More recently, there have also been many works on the fluctuations around the mean-field behavior. Grillakis, Margetis and Machedon proved the validity of Bogoliubov theory in a weak-coupling situation with mathematical rigor [19, 20, 17]. They showed that the number of excitations is finite in this case and that the convergence of the $N$-body wave function towards the respective solution of the Bogoliubov time evolution holds in $L^{2}$-norm. This implies also that the errors are small compared to the effects of Bogoliubov's theory. Their results have been generalized by many other authors in different situations [8, 18, 23, 26, 28, 31, 32, 33, 34, 37]. Corrections beyond the Bogoliubov dynamics were studied in [36, 5], including explicit formulas for higher contributions [6].

In parallel to these results concerning the dynamics, there are also numerous results related to the static question, which show closeness of the $N$-body ground state to the minimizer of an effective functional (see [25]). Bogoliubov theory has also been justified in this context [42, 16, 11, 43, 27, 38, 39, 40, 22, and allows for a resolution of the spectrum close to the ground state [11, 16, 42, 27, 3]. Recently, higher order corrections [7] and the interaction with a tracer particle [30] have also been considered in the static context.

\subsubsection{Heuristics of the mean-field scaling}

In this article we consider the mean-field scaling for the boson interactions, i.e. the situation where $g_{B}$ is of order $N^{-1}$. With a weak coupling of this form, we can expect the interaction energy of the $N$ bosons, which is roughly of order $N^{2} g_{B}$, to be of the same order as their kinetic energy. In models for dilute gases this is complemented by a scaling of the potential $V_{N}$ such that $V_{N}$ tends to $\delta$ weakly as $N \rightarrow \infty$. We will not consider this situation here. We only remark that, in addition to the difficulties in the derivation of the Bogoliubov Hamiltonian, it will be necessary to take into account the renormalization of the limiting model (see Remark 21).

As explained above, we are interested in a tracer particle entering the Bose gas. We want to consider a scaling, respectively coupling, which is such that the interaction of the tracer is with the excitations from the ground state. Since the vast majority of the bosons will be part of the condensate, the leading contribution to the energy of the tracer will generally come 
from the condensate and this should be of order $g_{I} N_{0} \sim g_{I} N$, where $N_{0}$ is the number of bosons in the condensate. However, since the condensate density is assumed to be constant, this contribution will be trivial, i.e. a constant. The next order would be the back-reaction on the tracer of small changes to the condensate, i.e. excitations. Since this is a second order effect one can expect it to be of order $g_{I}^{2} N$. We thus assume that $g_{I}$ is of order $N^{-1 / 2}$, which will also ensure that the number of excitations coming from the interaction with the tracer is of the same order as the number of excitations we have without tracer particle (i.e. of order one). This is exactly the same scaling as in [30].

The articles [10, 9] considered the different situation where the interaction of the tracer with the Bose gas is such that the density of the gas is changed significantly. To achieve this, the coupling constant between tracer and gas is chosen to be one. Thus the back-reaction of the gas particles and the tracer will be of order $N$ (respectively of the order of the density if the gas has large volume). In order to have a nice limiting regime the mass of the tracer-particle in [10, 9] was assumed to be of order $N$, resulting in an acceleration of order one.

\subsubsection{Heuristics of Bogoliubov theory}

We now briefly explain the heuristic derivation of the Bogoliubov-Fröhlich Hamiltonian (see also [21, Sect.3.3]), which we will subsequently make rigorous. Let $\varphi_{0} \equiv 1$ denote the constant function on the torus, which we think of as the condensate state, and extend this to a complete orthogonal system $\left(\varphi_{j}\right)_{j \in \mathbb{N}_{0}}$ of the one-particle Hilbert space $L^{2}\left(T^{d}\right)$ (of course, one can take the $\varphi_{j}$ to be plane waves, but for our purposes this is not necessary and we prefer to retain this generality). Let $a_{j}^{*}:=a^{*}\left(\varphi_{j}\right)$ and $a_{j}:=a\left(\varphi_{j}\right)$ be the creation, respectively annihilation, operators for the state $\varphi_{j}$ (see [41, Sect.X.7] for definitions of these, and related, objects).

The boson-Hamiltonian with coupling $g_{B}=N^{-1}$ can then be written as

$$
H_{B}=d \Gamma(-\Delta)+N^{-1} \sum_{j, k, \ell, m=0}^{\infty} V_{j k l m} a_{j}^{*} a_{k}^{*} a_{\ell} a_{m}
$$

with

$$
V_{j k \ell m}=\int_{T^{d}} \int_{T^{d}} \bar{\varphi}_{j}(y) \bar{\varphi}_{k}\left(y^{\prime}\right) V\left(y-y^{\prime}\right) \varphi_{\ell}\left(y^{\prime}\right) \varphi_{m}(y) d y^{\prime} d y .
$$

Bogoliubov's idea [4] is that, since the vast majority of particles will be in the condensate state, the operators $a_{0}^{*}$ and $a_{0}$ will give contributions of order $\sqrt{N}$, whereas $a_{j}^{*}$ and $a_{j}$ with $j \geq 1$ give contributions of order one. Consequently, all terms with less than two of the indices $j, k, \ell, m$ equal to zero are small. Replacing then $a_{0}^{*}$ and $a_{0}$ by $\sqrt{N}$ in the remaining ones and assuming that $V$ is even and $\int_{T^{d}} V=0$ (which corresponds to a constant shift in energy and eliminates all terms where more than two of the indices $j, k, \ell$ and $m$ are equal to zero - see also Lemma 1.1 below), one arrives at the Bogoliubov Hamiltonian

$$
H^{\mathrm{Bog}}=d \Gamma(-\Delta)+2 \sum_{j, k=1}^{\infty} V_{j 0 k 0} a_{j}^{*} a_{k}+\sum_{j, k=1}^{\infty} V_{j k 00} a_{j}^{*} a_{k}^{*}+\sum_{j, k=1}^{\infty} V_{00 j k} a_{j} a_{k} .
$$


If we apply the same reasoning to the interaction of the bosons with the tracer, we write

$$
\sum_{n=1}^{N} W\left(x-y_{n}\right)=\sum_{j, k=0}^{\infty} a_{j}^{*} a_{k}\left(W_{x}\right)_{j, k}
$$

with $W_{x}(y)=W(x-y),\left(W_{x}\right)_{j, k}=\int \bar{\varphi}_{j}(y) \varphi_{k}(y) W_{x}(y) d y$. Using that $\left(W_{x}\right)_{0,0}(x)=\int W=0$, and then setting $g_{I}=N^{-1 / 2}$, dropping the terms with $j \neq 0 \neq k$ and replacing $a_{0}^{*}$ and $a_{0}$ by $\sqrt{N}$ as above, we arrive at (see also Lemma 1.2 below)

$$
\sum_{j=1}^{\infty}\left(a_{j}\left(W_{x}\right)_{0, j}+a_{j}^{*}\left(W_{x}\right)_{j, 0}\right)=a\left(W_{x}\right)+a^{*}\left(W_{x}\right) .
$$

Adding this interaction as well as the kinetic energy of the tracer to the Bogoliubov Hamiltonian, we obtain the Bogoliubov-Fröhlich Hamiltonian

$$
H^{\mathrm{BF}}=-\frac{1}{2 m} \Delta_{x}+H^{\mathrm{Bog}}+a^{*}\left(W_{x}\right)+a\left(W_{x}\right) .
$$

Remark 1. An important feature of the original Bogoliubov Hamiltonian is that it is a quadratic expression in the operators $a, a^{*}$. There is a unitary Bogoliubov transformation $U_{\text {Bog }}$ (that amounts to changing the creation/annihilation operators) such that

$$
U_{\mathrm{Bog}} H^{\mathrm{Bog}} U_{\mathrm{Bog}}^{*}=d \Gamma(E)+E_{0}
$$

for a one-particle operator $E$ and a constant $E_{0}$. The excitations can thus be described by a non-interacting theory and, in the case of the torus, the energy levels can be computed explicitly (see e.g. [30]).

We emphasize that the Bogoliubov-Fröhlich Hamiltonian does not share this feature. Even though the expression (9) seems to be quadratic, the interaction depends on the position $x$ of the tracer particle, which also appears in the Laplacian. Performing an $x$-dependent Bogoliubov transformation will thus not yield a simple result (alternatively, introducing a field for the impurities and viewing $H^{\mathrm{BF}}$ as the restriction to the one-impurity space, one sees that the interaction is in fact cubic in the creation and annihilation operators, cf. [21, Sect.3.2]). One can, of course, transform $H^{\mathrm{BF}}$ using $U_{\mathrm{Bog}}$, which yields an operator of the form

$$
\left(1 \otimes U_{\mathrm{Bog}}\right) H^{\mathrm{BF}}\left(1 \otimes U_{\mathrm{Bog}}^{*}\right)=-\frac{1}{2 m} \Delta_{x}+d \Gamma(E)+a^{*}\left(\tilde{W}_{x}\right)+a\left(\tilde{W}_{x}\right)+E_{0},
$$

with a suitably transformed interaction $\tilde{W}$ (see [21, Sect.3.3] and [30, Eq.(1.10)] for explicit expressions). Operators of the form (11), with general $E$ and $W$, which feature the interaction of a particle (or several) with a quantum field by a linear coupling have been studied in many variants and are sometimes referred to as Fröhlich or polaron Hamiltonians. They include the original Fröhlich model [12] and the Nelson model [35]. One of the main motivations of the present work is to show rigorously that such a model arises as an effective description of a many-particle system. 
Remark 2. If, instead of the mean-field regime, we were to consider a dilute $N$-particle system (as in [2]) then, as an intermediate step, we would find a Hamiltonian of the same form as $H^{\mathrm{BF}}$, but with $W=W_{N}$ depending on $N$ and converging to $\delta$ weakly as $N \rightarrow \infty$. Since $a^{*}(\delta)$ is not a densely defined operator, it is not immediately clear how to define the limiting dynamics. It was shown in [24 that a renormalized version of $H^{\mathrm{BF}}$ with $W=\delta$ can be constructed in this case and that its unitary group can be approximated using operators with ultraviolet cut-off, up to a divergent phase. In a dilute system the particle number $N$ would function as an effective ultraviolet cutoff, so we expect that in this case the phase would have to be modified accordingly in order to obtain the effective dynamics generated by the renormalized Bogoliubov-Fröhlich Hamiltonian.

\subsection{The condensate-excitation representation}

In order to make the heuristics of the previous section rigorous, we will represent functions in $\mathcal{H}_{N}$ by decomposing them into their components along the (constant) condensate wavefunction $\varphi_{0}$ and in the orthogonal complement. The rigoros implementation of this idea was pioneered by Lewin, Nam, Serfaty and Solovej [27] and we closely follow their presentation. It naturally gives rise to Fock space and thus the representation of the excitations by a quantum field.

Recall that $\varphi_{0} \equiv 1$ denotes the constant function on $T^{d}$ and $\left(\varphi_{j}\right)_{j \in \mathbb{N}_{0}}$ a complete orthonormal system in $L^{2}\left(T^{d}\right)$ with $\varphi_{j} \in H^{2}\left(T^{d}\right), j \in \mathbb{N}$. Let

$$
P \psi=\int_{T^{d}} \psi(y) d y
$$

be the projection to $\operatorname{span}\left\{\varphi_{0}\right\}=: \mathfrak{H}_{0}$ in $L^{2}\left(T^{d}\right)$ and $Q=1-P$. The function $\varphi_{0}$ plays the role of the condensate wave-function, while $\mathfrak{H}_{+}:=Q L^{2}\left(T^{d}\right)$ is the Hilbert space of an excitation. The functions $\left(\varphi_{j}\right)_{j \in \mathbb{N}}$, with $j>0$, thus form a complete orthonormal system in $\mathfrak{H}_{+}$. Let $\Psi \in L^{2}\left(T^{d}\right)^{\otimes_{s} N}$, then we can write

$$
\Psi=(P+Q)^{\otimes N} \Psi=\sum_{j=0}^{N} \varphi_{0}^{\otimes(N-j)} \otimes_{s} \Phi^{(j)}
$$

with $\Phi^{(j)} \in \mathfrak{H}_{+}^{\otimes_{s} j}$. We can thus represent $\Psi \in L^{2}\left(T^{d}\right)^{\otimes_{s} N}$ uniquely by the sequence

$$
\left(\Phi^{(n)}\right)_{n \leq N} \in \bigoplus_{n=0}^{N} \mathfrak{H}_{+}^{\otimes_{s} n}=: \mathcal{F}_{+}^{\leq N} .
$$

We denote by $\mathcal{F}_{+}=\Gamma\left(\mathfrak{H}_{+}\right)$the Fock space of excitations, and by $\mathcal{F}_{+}^{\leq N}$ the truncated Fock space, defined as above.

The $N$-boson space $\mathcal{H}_{N}$ also contains an additional factor for the tracer particle. We define

$$
\mathcal{H}_{+}=: L^{2}\left(T^{d}\right) \otimes \mathcal{F}_{+}, \quad \mathcal{H}_{+}^{\leq N}=: L^{2}\left(T^{d}\right) \otimes \mathcal{F}_{+}^{\leq N} .
$$


Leaving the first tensor factor untouched, we obtain a unitary

$$
U_{N}: \mathcal{H}_{N} \rightarrow \mathcal{H}_{+}^{\leq N} \subset \mathcal{H}_{+} .
$$

By viewing $L^{2}\left(T^{d}\right)^{\otimes_{s} N}$ as embedded in the Fock space $\mathcal{F}=\Gamma\left(L^{2}\left(T^{d}\right)\right)$, the unitary can be expressed by the formula (see [27, Eq.(4.6)])

$$
U_{N} \Psi=\bigoplus_{j=0}^{N} Q^{\otimes j}\left(\frac{a_{0}^{N-j}}{\sqrt{(N-j) !}} \Psi\right) .
$$

Denote by $\mathcal{N}_{+}:=d \Gamma(Q)$ the number operator in $\mathcal{F}_{+}$. Then from (16) one easily deduces the following identities for $f, g \in \mathfrak{H}_{+}$(see [27, Prop.14]):

$$
\begin{aligned}
U_{N} a_{0}^{*} a_{0} U_{N}^{*} & =N-\mathcal{N}_{+} \\
U_{N} a^{*}(f) a_{0} U_{N}^{*} & =a^{*}(f) \sqrt{N-\mathcal{N}_{+}} \\
U_{N} a_{0}^{*} a(f) U_{N}^{*} & =\sqrt{N-\mathcal{N}_{+}} a(f) \\
U_{N} a(f)^{*} a(g) U_{N}^{*} & =a(f)^{*} a(g)
\end{aligned}
$$

Note that on the left hand side we only consider number-preserving combinations of creation and annihilation operators, so these expressions can be viewed as operators on $\mathcal{H}_{N}$.

We now apply this transformation to the interaction terms in the Hamiltonian.

Lemma 1.1. Let $\left(\varphi_{n}\right)_{n \in \mathbb{N}_{0}}$ be as above and assume that $V \in L^{2}\left(T^{d}\right)$ is an even function with $\int_{T^{d}} V(y) d y=0$ and relatively $\Delta$-bounded as an operator. The following identity holds in the sense of closed operators

$$
\begin{aligned}
& U_{N}\left(\sum_{j, k=1}^{N} V\left(y_{j}-y_{k}\right)\right) U_{N}^{*}=2 \sum_{j, k \in \mathbb{N}} V_{j 0 k 0} a_{j}^{*}\left(N-\mathcal{N}_{+}\right) a_{k} \\
& \quad+\sum_{j, \in \mathbb{N}} V_{j k 00} a_{j}^{*} \sqrt{N-\mathcal{N}_{+}} a_{k}^{*} \sqrt{N-\mathcal{N}_{+}}+\sum_{j, \in \mathbb{N}} V_{00 j k} \sqrt{N-\mathcal{N}_{+}} a_{j} \sqrt{N-\mathcal{N}_{+}} a_{k} \\
& \quad+2 \sum_{j, k, \ell \in \mathbb{N}}\left(V_{j k \ell 0} a_{j}^{*} \sqrt{N-\mathcal{N}_{+}} a_{k}^{*} a_{\ell}+V_{0 j k \ell} a_{j}^{*} a_{k} \sqrt{N-\mathcal{N}_{+}} a_{\ell}\right)+\sum_{j, k, \ell, m \in \mathbb{N}} V_{j k \ell m} a_{j}^{*} a_{k}^{*} a_{\ell} a_{m}
\end{aligned}
$$

with $V_{j k \ell m}$ defined by (5).

Proof. We write the mulitplication operator $\sum_{j, k} V\left(y_{j}-y_{k}\right)$ on $L^{2}\left(T^{d}\right)$ as in (4) (where $V_{j k \ell m}$ is well-defined since the $\varphi_{j} \in H^{2}$ and $V$ is $\Delta$-bounded). We then apply the transformation $U_{N}$ using the identities (17). Note that, since $V$ is even, $V_{0 j 0 k}=V_{j 0 k 0}$ are real. In order to transform the quartic expressions in $a_{j}, a_{j}^{*}$, these must be grouped into number-preserving pairs, e.g. for $j, k, \ell>0$,

$$
U_{N} a_{j}^{*} a_{k}^{*} a_{\ell} a_{0} U_{N}^{*}=U_{N} a_{j}^{*} a_{0} U_{N}^{*} U_{N} a_{k}^{*} a_{\ell} U_{N}^{*}=a_{j}^{*} \sqrt{N-\mathcal{N}_{+}} a_{k}^{*} a_{\ell} .
$$

The claim then follows by noting that $V_{j k \ell 0}=V_{k j 0 \ell}$ and that $\int V=0$ implies $V_{j 00 k}=0=V_{0 j k 0}$ for $j, k \in \mathbb{N}_{0}$. 
Lemma 1.2. Assume that $W \in L^{2}\left(T^{d}\right)$ be $\Delta$-bounded with $\int_{T^{d}} W(y) d y=0$ and set $W_{x}(y):=$ $W(x-y)$. The following identity holdsin the sense of closed operators

$$
U_{N}\left(\sum_{j=1}^{N} W\left(x-y_{j}\right)\right) U_{N}^{*}=\sqrt{N-\mathcal{N}_{+}} a\left(W_{x}\right)+a^{*}\left(W_{x}\right) \sqrt{N-\mathcal{N}_{+}}+d \Gamma\left(Q W_{x} Q\right) .
$$

Proof. We can rewrite $W_{x}$ as in (7). The claim then follows from the identities (17) by noting that

$$
\begin{aligned}
\left(W_{x}\right)_{00} & =P W_{x} P=\int_{T^{d}} W(x-y) d y=0 \\
\sum_{k=1}^{\infty}\left(W_{x}\right)_{k, 0} a_{k}^{*} & =\sum_{k=1}^{\infty} a^{*}\left(\varphi_{k}\right)\left\langle\varphi_{k}, W_{x} \varphi_{0}\right\rangle=a^{*}\left(Q W_{x} \varphi_{0}\right)=a^{*}\left(W_{x}\right), \\
\sum_{k, \ell=1}^{\infty} a_{k}^{*} a_{\ell}\left(W_{x}\right)_{k, \ell} & =d \Gamma\left(Q W_{x} Q\right) .
\end{aligned}
$$

\section{Main Result}

We assume that the $N$-particle initial state $\Psi_{N} \in \mathcal{H}_{N}$ is a member of a sequence such that

$$
\lim _{N \rightarrow \infty} U_{N} \Psi_{N}=: \Phi
$$

exists in $\mathcal{H}_{+}$. This assumption means that the number of excitations out of the condensate in the initial states $\Psi_{N}$ remains finite with probability one as $N \rightarrow \infty$. Note, however, that the expected number of excitations $\left\langle\mathcal{N}_{+} U_{N} \Psi_{N}, U_{N} \Psi_{N}\right\rangle$ may diverge.

Recall that the expression of $H^{\mathrm{BF}}$ in (9) is given by $H_{0}:=-\frac{1}{2 m} \Delta_{x}+d \Gamma(-\Delta)$ plus several terms that are linear or quadratic in creation and annihilation operators. If $V, W \in L^{2}\left(T^{d}\right)$, the latter are bounded relative to $\mathcal{N}_{+}$(see Proposition A.3). Consequently, $H^{\mathrm{BF}}$ is a well defined operator on $D\left(H_{0}\right)$, since on $\mathcal{F}_{+}$

$$
d \Gamma(-\Delta) \geq 4 \pi^{2} \mathcal{N}_{+}
$$

In fact, $H^{\mathrm{BF}}$ is essentially self-adjoint on $D\left(H_{0}\right)$, see Proposition A.3. Thus the time evolution

under $H^{\mathrm{BF}}, e^{-i H^{\mathrm{BF}} t} \Phi$, is well-defined and we may compare it to the time evolution with $H_{N}$ using $U_{N}$.

If we make the assumption that $\Phi \in D\left(H_{0}\right)$, then we obtain a quantitative estimate proving the closeness of the two evolutions for large $N$.

Theorem 2.1. Assume that $V, W \in L^{2}\left(T^{d}, \mathbb{R}\right)$ are infinitesimally bounded relative to $-\Delta$ and satisfy $V(-y)=V(y)$ and $\int_{T^{d}} V=0=\int_{T^{d}} W$. There exists $v>0$ such that for all $\Phi \in D\left(H_{0}\right)$ there is $K>0$ such that for all $N \in \mathbb{N}$ and $t \in \mathbb{R}$

$$
\left\|U_{N} e^{-i H_{N} t} U_{N}^{*} \Phi^{\leq N}-e^{-i H^{\mathrm{BF}} t} \Phi\right\|_{\mathcal{H}_{+}} \leq K e^{v|t|} N^{-1 / 4}
$$


The proof of Theorem 2.1 will be the content of the next section. Note that in this statement only $K$ depends on the intial states. By density of $D\left(H_{0}\right)$ in $\mathcal{H}_{+}$, the statement can be extended to any $\Phi \in \mathcal{H}_{+}$, but without an explicit rate of convergence.

Theorem 2.2. Assume that $V, W \in L^{2}\left(T^{d}, \mathbb{R}\right)$ are infinitesimally bounded relative to $-\Delta$ and satisfy $V(-y)=V(y)$ and $\int_{T^{d}} V=0=\int_{T^{d}} W$. Then for every $\Phi \in \mathcal{H}_{+}$and and every sequence $\Psi_{N}, N \in \mathbb{N}$, with $\lim _{N \rightarrow \infty} U_{N} \Psi_{N}=\Phi$ we have

$$
\lim _{N \rightarrow \infty}\left\|U_{N} e^{-i H_{N} t} \Psi_{N}-e^{-i H^{\mathrm{BF}} t} \Phi\right\|_{\mathcal{H}_{+}}=0
$$

uniformly in $t$ on compact subsets of $\mathbb{R}$.

Proof. This follows from Theorem 2.1 above by an approximation argument. Let $\Phi \in \mathcal{H}_{+}$be given and $T>0, \varepsilon>0$. Let $\widetilde{\Phi} \in D\left(H_{0}\right)$ with $\|\Phi-\widetilde{\Phi}\|_{\mathcal{H}_{+}}<\varepsilon$ and set $\widetilde{\Psi}_{N}=U_{N}^{*} \widetilde{\Phi} \leq N$. Since $\lim _{N \rightarrow \infty} U_{N} \Psi_{N}=\Phi$, we have for sufficiently large $N$

$$
\left\|\Psi_{N}-\widetilde{\Psi}_{N}\right\|_{\mathcal{H}_{N}} \leq\left\|U_{N} \Psi_{N}-\Phi\right\|_{\mathcal{H}_{+}}+\|\Phi-\widetilde{\Phi}\|+\left\|\widetilde{\Phi}^{>N}\right\|<3 \varepsilon
$$

By unitarity of the time evolutions we thus have

$$
\left\|U_{N} e^{-i H_{N} t} \Psi_{N}-e^{-i H^{\mathrm{BF}} t} \Phi\right\|_{\mathcal{H}_{+}}<4 \varepsilon+\left\|U_{N} e^{-i H_{N} t} \widetilde{\Psi}_{N}-e^{-i H^{\mathrm{BF}} t} \widetilde{\Phi}\right\|_{\mathcal{H}_{+}} .
$$

By Theorem 2.1 the last term is bounded for $|t| \leq T$ by

$$
\left\|U_{N} e^{-i H_{N} t} \widetilde{\Psi}_{N}-e^{-i H^{\mathrm{BF}} t} \widetilde{\Phi}\right\|_{\mathcal{H}_{+}} \leq K e^{v T} N^{-1 / 4}
$$

so it is smaller than $\varepsilon$ for sufficiently large $N$.

\section{Proof of convergence}

Throughout this section we assume the hypothesis of Theorem 2.1. As a fist step we will prove that the time evolution $U_{N} e^{-i H_{N} t} U_{N}^{*}$ is well approximated by the time evolution generated by a truncation of $U_{N} H_{N} U_{N}^{*}$, where terms that are more than quadratic in the creation and annihilation operators are neglected. We collect the leading-order terms in an auxiliary operator $H^{\text {aux }}$. This operator acts on $\mathcal{H}_{+}^{\leq N}$ and is naturally extended to $\mathcal{H}_{+}$by zero. Since $\left\langle\varphi_{k}, \Delta \varphi_{0}\right\rangle=0=\left\langle\varphi_{0}, \Delta \varphi_{k}\right\rangle$ for $k \in \mathbb{N}_{0}$, we have

$$
-U_{N} \sum_{j=1}^{N} \Delta_{y_{j}} U_{N}^{*}=d \Gamma^{\leq N}(-\Delta),
$$


where $d \Gamma^{\leq N}(-\Delta)$ denotes the projection of $d \Gamma(-\Delta)$ to $\mathcal{H}_{+}^{\leq N}$. In view of Lemmas 1.2, 1.1, we define $H_{N}^{\text {aux }}$ by the expression

$$
\begin{aligned}
H_{N}^{\text {aux }}:= & -\frac{1}{2 m} \Delta_{x}+d \Gamma^{\leq N}(-\Delta)+\frac{2}{N} \sum_{j, k>1} V_{j 0 k 0} a_{j}^{*}\left(N-\mathcal{N}_{+}\right) a_{k} \\
& +\frac{1}{N} \sum_{j, k>1} V_{j k 00} a_{j}^{*} \sqrt{N-\mathcal{N}_{+}} a_{k}^{*} \sqrt{N-\mathcal{N}_{+}} \\
& +\frac{1}{N} \sum_{j, k>1} V_{00 j k} \sqrt{N-\mathcal{N}_{+}} a_{k} \sqrt{N-\mathcal{N}_{+}} a_{k} \\
& +\frac{1}{\sqrt{N}} a^{*}\left(W_{x}\right) \sqrt{N-\mathcal{N}_{+}}+\frac{1}{\sqrt{N}} \sqrt{N-\mathcal{N}_{+}} a\left(W_{x}\right)
\end{aligned}
$$

on $D\left(H_{N}^{\text {aux }}\right)=U_{N} D\left(H_{N}\right) \subset \mathcal{H}_{+}^{\leq N}$. Note that $H_{N}^{\text {aux }}$ is self-adjoint by Lemma A.2 and that $H^{\mathrm{BF}}$ is obtained from $H_{N}^{\text {aux }}$ by simply replacing $\sqrt{1-\mathcal{N}_{+} / N}$ by one and extending to $\mathcal{H}_{+}$.

To prove closeness of $e^{-i H_{N}^{\text {aux }} t}$ and $U_{N} e^{-i H_{N} t} U_{N}^{*}$, and later $e^{-i H^{\mathrm{BF}} t}$ and $e^{-i H_{N}^{\text {aux }} t}$, the crucial ingredient is control of the number of excitations for all times.

Lemma 3.1. For any $\Phi \in D\left(H_{0}\right)$ set

$$
\begin{aligned}
\alpha(t) & :=\left\|\left(\mathcal{N}_{+}+1\right) U_{N} e^{-i H_{N} t} U_{N}^{*} \Phi^{\leq N}\right\|^{2} \\
\alpha^{\mathrm{aux}}(t) & :=\left\|\left(\mathcal{N}_{+}+1\right) e^{-i H_{N}^{\text {aux }} t} \Phi^{\leq N}\right\|^{2} \\
\alpha^{\mathrm{BF}}(t) & :=\left\|\left(\mathcal{N}_{+}+1\right) e^{-i H^{\mathrm{BF}} t} \Phi\right\|^{2} .
\end{aligned}
$$

There exists a constant $v$ such that for all $\Phi \in D\left(H_{0}\right), N \in \mathbb{N}$ and $t \in \mathbb{R}$ we have

$$
\alpha^{\bullet}(t) \leq \alpha^{\bullet}(0) e^{v|t|}
$$

for $\bullet \in\{\emptyset$, aux, BF $\}$.

Proof. Since $\Phi \in D\left(\mathcal{N}_{+}\right)$, the statement clearly holds for $t=0$. We will use Grönwall's Lemma to obtain a bound for all $t \geq 0$ (the proof for $t \leq 0$ is the same).

We first prove the claim for $\alpha$ and $\alpha^{\text {aux }}$ (since $H_{N}^{\text {aux }}$ is obtained from $U_{N} H_{N} U_{N}^{*}$ by dropping some terms the proof for $\alpha^{\text {aux }}$ is contained in the one fo $\alpha$ ). By Lemma A.2, we have $\Phi^{\leq N} \in$ $U_{N} D\left(H_{N}\right)=D\left(H^{\text {aux }}\right)$. Thus

$$
\begin{aligned}
\frac{d \alpha}{d t}(t) & =\frac{d}{d t}\left\langle U_{N} e^{-i H_{N} t} U_{N}^{*} \Phi^{\leq N},\left(\mathcal{N}_{+}+1\right)^{2} U_{N} e^{-i H_{N} t} U_{N}^{*} \Phi^{\leq N}\right\rangle \\
& =\left\langle U_{N} e^{-i H_{N} t} U_{N}^{*} \Phi^{\leq N}, i\left[U_{N} H_{N} U_{N}^{*},\left(\mathcal{N}_{+}+1\right)^{2}\right] U_{N} e^{-i H_{N} t} U_{N}^{*} \Phi^{\leq N}\right\rangle .
\end{aligned}
$$

With $\left[U_{N} H_{N} U_{N}^{*},\left(\mathcal{N}_{+}+1\right)^{2}\right]=\left(\mathcal{N}_{+}+1\right)\left[U_{N} H_{N} U_{N}^{*}, \mathcal{N}_{+}\right]+\left[U_{N} H_{N} U_{N}^{*}, \mathcal{N}_{+}\right]\left(\mathcal{N}_{+}+1\right)$ and CauchySchwarz we obtain

$$
\left|\frac{d \alpha}{d t}(t)\right| \leq 2 \sqrt{\alpha(t)}\left\|\left[U_{N} H_{N} U_{N}^{*}, \mathcal{N}_{+}\right] e^{-i H_{N} t} U_{N}^{*} \Phi^{\leq N}\right\| .
$$


We can thus prove the claim by bounding the latter norm in terms of $\sqrt{\alpha}$.

To do this, we start with the terms from $H_{N}^{\text {aux }}$. Using that

$$
\begin{aligned}
& {\left[a_{j}^{*} a_{j}, a_{k}^{*} a_{\ell}^{*}\right]=\left(\delta_{j k}+\delta_{j \ell}\right) a_{j}^{*} a_{j}^{*}} \\
& {\left[a_{j}^{*} a_{j}, a_{k} a_{\ell}\right]=-\left(\delta_{j k}+\delta_{j \ell}\right) a_{j} a_{j}}
\end{aligned}
$$

and $\mathcal{N}_{+}=\sum_{j=1}^{\infty} a_{j}^{*} a_{j}$ we find

$$
\begin{aligned}
& {\left[\mathcal{N}_{+}, \sum_{j, k=1}^{\infty} V_{j k 00} a_{j}^{*} a_{k}^{*}\right]=2 \sum_{j, k=1}^{\infty} V_{j k 00} a_{j}^{*} a_{k}^{*}} \\
& {\left[\mathcal{N}_{+}, \sum_{j, k=1}^{\infty} V_{00 j k} a_{j} a_{k}\right]=-2 \sum_{j, k=1}^{\infty} V_{00 j k} a_{j} a_{k} .}
\end{aligned}
$$

We thus have

$$
\begin{aligned}
{\left[\mathcal{N}_{+}, H_{N}^{\mathrm{aux}}\right]=} & \frac{2}{N} \sum_{j, k=1}^{\infty} V_{j k 00} a_{j}^{*} \sqrt{N-\mathcal{N}_{+}} a_{k}^{*} \sqrt{N-\mathcal{N}_{+}} \\
& -\frac{2}{N} \sum_{j, k=1}^{\infty} V_{00 j k} \sqrt{N-\mathcal{N}_{+}} a_{k} \sqrt{N-\mathcal{N}_{+}} a_{k} \\
& +\frac{1}{\sqrt{N}} a^{*}\left(W_{x}\right) \sqrt{N-\mathcal{N}_{+}}-\frac{1}{\sqrt{N}} \sqrt{N-\mathcal{N}_{+}} a\left(W_{x}\right) .
\end{aligned}
$$

As $\left\|N^{-1 / 2} \sqrt{N-\mathcal{N}_{+}}\right\|_{\mathcal{H}_{+}^{\leq N} \rightarrow \mathcal{H}_{+}^{\leq N}} \leq 1$, this operator is bounded relative to $\mathcal{N}_{+}$by Lemma A.1 and (60), which gives the desired bound. The expression for $U_{N} H_{N} U_{N}^{*}$ additionally contains the term $N^{-1 / 2} d \Gamma\left(Q W_{x} Q\right)$ from the interaction (see Lemma 1.2), which commutes with $\mathcal{N}_{+}$. Additionally, there are the terms from the boson interaction (see Lemma 1.1)

$$
\begin{aligned}
& \frac{2}{N} \sum_{j, k, \ell=1}^{\infty}\left(V_{j k \ell 0} a_{j}^{*} \sqrt{N-\mathcal{N}_{+}} a_{k}^{*} a_{\ell}+V_{0 j k \ell} a_{j}^{*} a_{k} \sqrt{N-\mathcal{N}_{+}} a_{\ell}\right), \\
& \frac{1}{N} \sum_{j, k, \ell, m=1}^{\infty} V_{j k \ell m} a_{j}^{*} a_{k}^{*} a_{\ell} a_{m} .
\end{aligned}
$$

The latter also commutes with $\mathcal{N}_{+}$, so it remains to bound the commutator of $\mathcal{N}_{+}$with the first line. We have from the canonical commutation relations (for the first term - the second one yields minus the adjoint)

$$
\left[\mathcal{N}_{+}, \frac{2}{N} V_{j k \ell 0} a_{j}^{*} \sqrt{N-\mathcal{N}_{+}} a_{k}^{*} a_{\ell}\right]=-\frac{2}{N} \sum_{j, k, \ell=1}^{\infty} V_{j k \ell 0} a_{j}^{*} \sqrt{N-\mathcal{N}_{+}} a_{k}^{*} a_{\ell} .
$$


In order to bound this operator, let $\Psi, \Xi \in \mathcal{H}_{+}^{\leq N}$, and rewrite

$$
\begin{aligned}
& \sum_{j, k, \ell=1}^{\infty}\left\langle\Psi, V_{j k \ell 0} a_{j}^{*} \sqrt{N-\mathcal{N}_{+}} a_{k}^{*} a_{\ell} \Xi\right\rangle \\
& =\int_{\left(T^{d}\right)^{2}} \sum_{j, k, \ell=1}^{\infty} \bar{\varphi}_{j}(y) \varphi_{k}\left(y^{\prime}\right)\left\langle\sqrt{N-\mathcal{N}_{+}} a_{j} a_{k} \Psi, V\left(y-y^{\prime}\right) \varphi_{\ell}(y) a_{\ell} \Xi\right\rangle d y d y^{\prime} .
\end{aligned}
$$

By the Cauchy-Schwarz inequality and the fact that $\left\|N^{-1 / 2} \sqrt{N-\mathcal{N}_{+}}\right\|_{\mathcal{H}_{+}^{\leq N} \rightarrow \mathcal{H}_{+}^{\leq N}} \leq 1$ we thus have

$$
\begin{aligned}
\frac{1}{N}\left|\sum_{j, k, \ell=1}^{\infty}\left\langle\Psi, V_{j k \ell 0} a_{j}^{*} \sqrt{N-\mathcal{N}_{+}} a_{k}^{*} a_{\ell} \Xi\right\rangle\right| \leq & \left(\int_{\left(T^{d}\right)^{2}}\left\|\sum_{j, k=1}^{\infty} \varphi_{j}(y) \varphi_{k}\left(y^{\prime}\right) a_{j} a_{k} \Psi\right\|^{2} d y d y^{\prime}\right)^{1 / 2} \\
& \left(\int_{\left(T^{d}\right)^{2}}\left\|N^{-1} \sum_{\ell=1}^{\infty} V\left(y-y^{\prime}\right) \varphi_{\ell}(y) a_{\ell} \Xi\right\|^{2} d y d y^{\prime}\right)^{1 / 2} .
\end{aligned}
$$

Now

$$
\begin{aligned}
& N^{-1} \int_{\left(T^{d}\right)^{2}}\left\|\sum_{\ell=1}^{\infty} V\left(y-y^{\prime}\right) \varphi_{\ell}(y) a_{\ell} \Xi\right\|^{2} d y d y^{\prime} \\
& \leq \sup _{y \in T^{d}}\left\{\int_{T^{d}} V^{2}\left(y-y^{\prime}\right) d y^{\prime}\right\} N^{-1} \int_{T^{d}}\left\|\sum_{\ell=1}^{\infty} \varphi_{\ell}(y) a_{\ell} \Xi\right\|^{2} d y \\
& =\sup _{y \in T^{d}}\left\{\int_{T^{d}} V^{2}\left(y-y^{\prime}\right) d y^{\prime}\right\} N^{-1} \int_{T^{d}} \sum_{\ell, m=1}^{\infty}\left\langle\varphi_{\ell}(y) a_{\ell} \Xi, \varphi_{m}(y) a_{m} \Xi\right\rangle d y \\
& \leq N^{-1}\|V\|_{L^{2}\left(T^{d}\right)}^{2} \sum_{\ell=1}^{\infty}\left\langle a_{\ell} \Xi, a_{\ell} \Xi\right\rangle \leq\|V\|_{L^{2}\left(T^{d}\right)}^{2}\|\Xi\|^{2}
\end{aligned}
$$

where we used that the $\left(\varphi_{n}\right)_{n \in \mathbb{N}}$ form an ONB and that $\sum_{k \in \mathbb{N}} a_{k}^{*} a_{k}=\mathcal{N}_{+}$. Similarly,

$$
\begin{aligned}
& \int_{\left(T^{d}\right)^{2}}\left\|\sum_{j, k=1}^{\infty} \varphi_{j}(y) \varphi_{k}\left(y^{\prime}\right) a_{j} a_{k} \Psi\right\|^{2} d y d y^{\prime} \\
& =\int_{\left(T^{d}\right)^{2}}\left\langle\sum_{j, k, \ell, m=1}^{\infty} \varphi_{j}(y) \varphi_{k}\left(y^{\prime}\right) a_{j} a_{k} \Psi, \varphi_{\ell}(y) \varphi_{m}\left(y^{\prime}\right) a_{\ell} a_{m} \Psi\right\rangle d y d y^{\prime} \\
& =\left\langle\sum_{j, k=1}^{\infty} a_{j} a_{k} \Psi, a_{j} a_{k} \Psi\right\rangle
\end{aligned}
$$

and we have

$$
\begin{aligned}
\sum_{j, k=1}^{\infty}\left\langle a_{j} a_{k} \Psi, a_{j} a_{k} \Psi\right\rangle & =\sum_{j, k=1}^{\infty}\left\langle\Psi,\left(a_{j}^{*} a_{j} a_{k}^{*} a_{k}+a_{j}^{*}\left[a_{k}^{*}, a_{j}\right] a_{k}\right) \Psi\right\rangle \\
& =\left\langle\Psi, \mathcal{N}_{+}\left(\mathcal{N}_{+}-1\right) \Psi\right\rangle \leq\left\|\mathcal{N}_{+} \Psi\right\|^{2}
\end{aligned}
$$


This implies that the commutator (36) is $\mathcal{N}_{+}$-bounded uniformly in $N$, so, by our earlier reasoning, there is a constant $C$ such that

$$
\frac{d \alpha}{d t}(t) \leq\left|\frac{d \alpha}{d t}(t)\right| \leq C \alpha(t)
$$

Thus by Grönwall's Lemma $\alpha(t) \leq \alpha(0) e^{C t}$, which proves the claim for $\alpha, \alpha^{\text {aux }}$.

The proof for $\alpha^{\mathrm{BF}}$ follows from the same argument, since $\left[\mathcal{N}_{+}, H^{\mathrm{BF}}\right]$ is $\mathcal{N}_{+}$-bounded by the identities (31), (32) and Lemma A.1. Taking the growth rate $v$ to be the maximum of the constants for $\alpha, \alpha^{\mathrm{BF}}$ proves the claim.

\subsection{Proof of Theorem 2.1}

Lemma 3.2. Let $\mathcal{H}$ be a Hilbert space and $D \subset \mathcal{H}$ a dense subspace. If $\left(H_{1}, D\right),\left(H_{2}, D\right)$ are self-adjoint operators, then for $\Psi_{j} \in D, j=1,2$, and $t \geq 0$

$$
\left\|e^{-i H_{1} t} \Psi_{1}-e^{-i H_{2} t} \Psi_{2}\right\|_{\mathcal{H}}^{2} \leq\left\|\Psi_{1}-\Psi_{2}\right\|_{\mathcal{H}}^{2}+2 \int_{0}^{t}\left|\left\langle e^{-i H_{1} s} \Psi_{1},\left(H_{1}-H_{2}\right) e^{-i H_{2} 2} \Psi_{2}\right\rangle\right| d s .
$$

Proof. We have

$$
\frac{d}{d t}\left\|e^{-i H_{1} t} \Psi_{1}-e^{-i H_{2} t} \Psi_{2}\right\|_{\mathcal{H}}^{2}=-2 \operatorname{Re}\left\langle e^{-i H_{1} t} \Psi_{1}, i\left(H_{1}-H_{2}\right) e^{-i H_{2} t} \Psi_{2}\right\rangle,
$$

so the claim follows from the fundamental theorem of calculus.

Lemma 3.3. Assume the hypothesis of Theorem [2.1 and let $\Phi \in D\left(H_{0}\right), \Psi_{N}=U_{N}^{*} \Phi^{\leq N}$. There exists $K>0$ such that for all $N \in \mathbb{N}$ and $t \in \mathbb{R}$

$$
\left\|e^{-i H_{N} t} \Psi_{N}-U_{N}^{*} e^{-i H_{N}^{\text {aux }} t} U_{N} \Psi_{N}\right\| \leq K N^{-1 / 4} e^{v|t|},
$$

where $v$ is the constant of Lemma 3.1.

Proof. By Lemma A.2, we have $D\left(H_{0}\right) \subset D\left(H^{\text {aux }}\right)$ and thus $\Psi_{N} \in D\left(H_{N}\right)=U_{N}^{*} D\left(H^{\text {aux }}\right)$. Using Lemma 3.2 it then follows that

$$
\begin{aligned}
& \left\|e^{-i H_{N} t} \Psi_{N}-U_{N}^{*} e^{-i H_{N}^{\text {aux }} t} U_{N} \Psi_{N}\right\|^{2} \\
& \quad \leq 2 \int_{0}^{t}\left|\left\langle e^{-i H_{N} t} \Psi_{N},\left(H_{N}-U_{N}^{*} H^{\text {aux }} U_{N}\right) U_{N}^{*} e^{-i H_{N}^{\text {aux }} t} U_{N} \Psi_{N}\right\rangle\right| d s \\
& \quad=2 \int_{0}^{t}\left|\left\langle U_{N} e^{-i H_{N} t} \Psi_{N},\left(U_{N} H_{N} U_{N}^{*}-H^{\text {aux }}\right) e^{-i H_{N}^{\text {aux }} t} \Phi^{\leq N}\right\rangle\right| d s .
\end{aligned}
$$

In view of Lemmas 1.2, 1.1, we have

$$
\begin{aligned}
U_{N} H_{N} U_{N}^{*}-H^{\text {aux }}= & \frac{1}{\sqrt{N}} d \Gamma\left(Q W_{x} Q\right) \\
& +\frac{2}{N} \sum_{j, k, \ell=1}^{\infty}\left(V_{j k \ell 0} a_{j}^{*} \sqrt{N-\mathcal{N}_{+}} a_{k}^{*} a_{\ell}+V_{0 j k \ell} a_{j}^{*} a_{k} \sqrt{N-\mathcal{N}_{+}} a_{\ell}\right) \\
& +\frac{1}{N} \sum_{j, k, \ell, m=1}^{\infty} V_{j k \ell m} a_{j}^{*} a_{k}^{*} a_{\ell} a_{m} .
\end{aligned}
$$


The first term in (45) satisfies, by the reasoning of (38), (39) and (40)

$$
\begin{aligned}
& \frac{2}{N}\left|\sum_{j, k, \ell=1}^{\infty}\left\langle U_{N} e^{-i H_{N} t} \Psi_{N}, V_{j k \ell 0} a_{j}^{*} \sqrt{N-\mathcal{N}_{+}} a_{k}^{*} a_{\ell} e^{-i H_{N}^{\text {aux }} t} \Phi^{\leq N}\right\rangle\right| \\
& \quad \leq \frac{2}{\sqrt{N}}\|V\|_{L^{2}}\left\|\sqrt{\mathcal{N}_{+}} U_{N} e^{-i H_{N} t} \Psi_{N}\right\|\left\|\mathcal{N}_{+} e^{-i H_{N}^{\text {aux }}} \Phi^{\leq N}\right\| \\
& \quad \leq \frac{2}{\sqrt{N}}\|V\|_{L^{2}}\left(\alpha(t) \alpha^{\operatorname{aux}}(t)\right)^{1 / 2} .
\end{aligned}
$$

The adjoint term from (45) satisfies the same bound.

The quartic term (46) will require some regularity of $\Phi$ (unless $V \in L^{\infty}$ ). First, we may expand

$$
\begin{aligned}
& \sum_{j, k, \ell, m=1}^{\infty}\left\langle U_{N} e^{-i H_{N} t} \Psi_{N}, V_{j k \ell m} a_{j}^{*} a_{k}^{*} a_{\ell} a_{m} e^{-i H_{N}^{\text {aux }} t} \Phi^{\leq N}\right\rangle \\
= & \int_{\left(T^{d}\right)^{2}} \sum_{j, k, \ell, m=1}^{\infty}\left\langle\varphi_{j}(y) \varphi_{k}\left(y^{\prime}\right) a_{j} a_{k} U_{N} e^{-i H_{N} t} \Psi_{N}, V\left(y-y^{\prime}\right) \varphi_{\ell}(y) \varphi_{m}\left(y^{\prime}\right) a_{\ell} a_{m} e^{-i H_{N}^{\text {aux }} t} \Phi^{\leq N}\right\rangle d y d y^{\prime} .
\end{aligned}
$$

Then, noting that the Laplacian is self-adjoint and invertible on $\mathfrak{H}_{+}$, multiplying with the identity operator in the form $\Delta_{y}^{-1} \Delta_{y}$ and using the Cauchy-Schwarz inequality gives

$$
\begin{gathered}
\frac{1}{N}\left|\sum_{j, k, \ell, m=1}^{\infty}\left\langle U_{N} e^{-i H_{N} t} \Psi_{N}, V_{j k \ell m} a_{j}^{*} a_{k}^{*} a_{\ell} a_{m} e^{-i H_{N}^{\text {aux }} t} \Phi^{\leq N}\right\rangle\right| \\
\leq\left(\int_{\left(T^{d}\right)^{2}} \frac{1}{N}\left\|\Delta_{y}^{-1} V\left(y-y^{\prime}\right) \sum_{j, k=1}^{\infty} \varphi_{j}(y) \varphi_{k}\left(y^{\prime}\right) a_{j} a_{k} U_{N} e^{-i H_{N} t} \Psi_{N}\right\|^{2} d y d y^{\prime}\right)^{1 / 2} \\
\quad \times\left(\int_{\left(T^{d}\right)^{2}} \frac{1}{N}\left\|\Delta_{y} \sum_{\ell, m=1}^{\infty} \varphi_{\ell}(y) \varphi_{m}\left(y^{\prime}\right) a_{\ell} a_{m} e^{-i H_{N}^{\text {aux }} t} \Phi^{\leq N}\right\|^{2} d y d y^{\prime}\right)^{1 / 2} .
\end{gathered}
$$

Using (40), (41), we have

$$
\text { (49) } \leq\left\|\Delta^{-1} V\right\|_{L^{2} \rightarrow L^{2}} N^{-1 / 2}\left\|\mathcal{N}_{+} U_{N} e^{-i H_{N} t} \Psi_{N}\right\| \leq C N^{-1 / 2} \alpha(t)^{1 / 2},
$$

since $\Delta^{-1} V$ is a bounded operator by hypothesis.

For (50) we use that $\left\|\mathcal{N}_{+} e^{-i H_{N}^{\text {aux }} t} \Phi^{\leq N}\right\| \leq N\left\|e^{-i H_{N}^{\text {aux }} t} \Phi^{\leq N}\right\|$ to obtain, similarly to (40),

$$
\begin{aligned}
\text { (50) } & \leq\left(\int_{T^{d}} \sum_{\ell, m=1}^{\infty}\left\langle\Delta_{y} \sum_{\ell=1}^{\infty} \varphi_{\ell}(y) a_{\ell} e^{-i H_{N}^{\text {aux }} t} \Phi^{\leq N}, \Delta_{y} \sum_{m=1}^{\infty} \varphi_{m}(y) a_{m} e^{-i H_{N}^{\text {aux }} t} \Phi^{\leq N}\right\rangle d y\right)^{1 / 2} \\
& =\left\langle e^{-i H_{N}^{\text {aux }} t} \Phi^{\leq N}, d \Gamma\left(\Delta^{2}\right) e^{-i H_{N}^{\text {aux }} t} \Phi^{\leq N}\right\rangle^{1 / 2} \\
& \leq\left\|d \Gamma(-\Delta) e^{-i H_{N}^{\text {aux }} t} \Phi^{\leq N}\right\| .
\end{aligned}
$$


Now

$\left\|d \Gamma(-\Delta) e^{-i H_{N}^{\text {aux }} t} \Phi^{\leq N}\right\| \leq\left\|H_{0} e^{-i H_{N}^{\text {aux }} t} \Phi^{\leq N}\right\| \leq\left\|e^{-i H_{N}^{\text {aux }} t} H_{N}^{\text {aux }} \Phi^{\leq N}\right\|+\left\|\left(H_{N}^{\text {aux }}-H_{0}\right) e^{-i H_{N}^{\text {aux }} t} \Phi^{\leq N}\right\|$,

and the difference $H_{N}^{\text {aux }}-H_{0}$ is a quadratic operator that is $\mathcal{N}_{+}$-bounded uniformly in $N$ by Lemma A.1 and (60). We thus have the following bound on the quartic term (46)

$\frac{2}{N}\left|\sum_{j, k, \ell, m=1}^{\infty}\left\langle U_{N} e^{-i H_{N} t} \Psi_{N}, V_{j k \ell m} a_{j}^{*} a_{k}^{*} a_{\ell} a_{m} e^{-i H_{N}^{\mathrm{aux} t}} \Phi^{\leq N}\right\rangle\right| \leq C N^{-1 / 2} \sqrt{\alpha(t)}\left(\left\|H_{N}^{\text {aux }} \Phi^{\leq N}\right\|+\sqrt{\alpha^{\text {aux }}(t)}\right)$.

By a similar argument (see also (61)), we have the bound

$$
\begin{aligned}
& \frac{1}{\sqrt{N}}\left|\left\langle U_{N} e^{-i H_{N} t} \Psi_{N}, d \Gamma\left(Q W_{x} Q\right) e^{-i H_{N}^{\text {aux }} t} \Phi^{\leq N}\right\rangle\right| \\
& \leq \sup _{x \in T^{d}}\left\|\Delta^{-1} W_{x}\right\|_{L^{2} \rightarrow L^{2}} N^{-1 / 2}\left\|\mathcal{N}_{+}^{1 / 2} U_{N} e^{-i H_{N} t} \Psi_{N}\right\|\left\|d \Gamma(-\Delta) e^{-i H_{N}^{\text {aux }} t} \Phi^{\leq N}\right\| \\
& \leq C N^{-1 / 2} \sqrt{\alpha(t)}\left(\left\|H_{N}^{\operatorname{aux}} \Phi^{\leq N}\right\|+\sqrt{\alpha^{\operatorname{aux}}(t)}\right) .
\end{aligned}
$$

Using the assumption that $\Phi \in D\left(H_{0}\right) \subset D\left(H^{\text {aux }}\right)$, Lemma 3.1 and integrating in (43) yields the claim.

To complete the proof of Theorem 2.1, it remains to prove the following Lemma on the approximation of $e^{-i H_{N}^{\mathrm{aux}} t}$ by $e^{-i H^{\mathrm{BF}} t}$, which essentially amounts to removing the restriction to $\mathcal{H}_{+}^{\leq N}$.

Lemma 3.4. Assume the hypothesis of Theorem 2.1 and let $\Phi \in D\left(H_{0}\right)$. There exists a constant $K$ such that for all $N \in \mathbb{N}$ and $t \in \mathbb{R}$

$$
\left\|e^{-i H_{N}^{\mathrm{aux}} t} \Phi^{\leq N}-e^{-i H^{\mathrm{BF}} t} \Phi\right\| \leq K e^{v|t|} N^{-1 / 4},
$$

where $v$ is the constant of Lemma 3.1.

Proof. We have $\Phi \in D\left(H_{0}\right) \subset D\left(H^{\mathrm{BF}}\right)$ (see Proposition A.3), so $\Phi^{\leq N} \in D\left(H_{N}^{\text {aux }}\right)$ by Lemma A.2. Note that

$$
\left\|\Phi-\Phi^{\leq N}\right\|^{2}=\sum_{n=N+1}^{\infty}\left\|\Phi^{(n)}\right\|^{2} \leq(N+1)^{-2}\left\|\mathcal{N}_{+} \Phi\right\|^{2},
$$

so, by Lemma 3.2, we have

$$
\left\|e^{-i H_{N}^{\mathrm{anx}} t} \Phi^{\leq N}-e^{-i H^{\mathrm{BF}} t} \Phi\right\|^{2} \leq(N+1)^{-2}\left\|\mathcal{N}_{+} \Phi\right\|^{2}+\int_{0}^{t}\left|\left\langle e^{-i H_{N}^{\mathrm{aux}}} \Phi^{\leq N},\left(H_{N}^{\mathrm{aux}}-H^{\mathrm{BF}}\right) e^{-i H^{\mathrm{BF}} s} \Phi\right\rangle\right| d s .
$$


The difference of the operators can be written as

$$
\begin{aligned}
H_{N}^{\mathrm{aux}}-H^{\mathrm{BF}}= & -\frac{2}{N} \sum_{j, k>1} V_{j 0 k 0} a_{j}^{*} \mathcal{N}_{+} a_{k} \\
& +\frac{1}{N} \sum_{j, k>1} V_{j k 00} a_{j}^{*}\left(\sqrt{N-\mathcal{N}_{+}}-\sqrt{N}\right) a_{k}^{*}\left(\sqrt{N-\mathcal{N}_{+}}-\sqrt{N}\right) \\
& +\frac{1}{N} \sum_{j, k>1} V_{00 j k}\left(\sqrt{N-\mathcal{N}_{+}}-\sqrt{N}\right) a_{k}\left(\sqrt{N-\mathcal{N}_{+}}-\sqrt{N}\right) a_{k} \\
& +\frac{1}{\sqrt{N}} a^{*}\left(W_{x}\right)\left(\sqrt{N-\mathcal{N}_{+}}-\sqrt{N}\right)+\frac{1}{\sqrt{N}}\left(\sqrt{N-\mathcal{N}_{+}}-\sqrt{N}\right) a\left(W_{x}\right) .
\end{aligned}
$$

Using that

$$
\begin{aligned}
a_{k}^{*} \mathcal{N}_{+} & =\left(\mathcal{N}_{+}-1\right) a_{k}^{*} \\
a_{k}^{*} \sqrt{N-\mathcal{N}_{+}} & =\sqrt{N+1-\mathcal{N}_{+}} a_{k}^{*} \\
a_{k} \sqrt{N-\mathcal{N}_{+}} & =\sqrt{N-\mathcal{N}_{+}-1} a_{k}
\end{aligned}
$$

we can move all of the $\mathcal{N}_{+}$-dependent factors to the left. The factors on the right are then terms that also appear in $H^{\mathrm{BF}}$ and are $\mathcal{N}_{+}$-bounded by Lemma A.1 and (60). With

$$
\begin{aligned}
N^{-1} & \left\|\left(\sqrt{N+1-\mathcal{N}_{+}} \sqrt{N+2-\mathcal{N}_{+}}-N\right) \Psi\right\|^{2} \\
& \leq N^{-1}\left\langle\left((N+2)^{2}+N^{2}\right) \Psi, \Psi\right\rangle-2 N^{-1} \operatorname{Re}\left\langle N\left(N+1-\mathcal{N}_{+}\right) \Psi, \Psi\right\rangle \\
& \leq 2 N^{-1}\langle(2 N+16) \Psi, \Psi\rangle+2\left\langle\Psi, \mathcal{N}_{+} \Psi\right\rangle \leq C\left(\|\Psi\|+\left\|\mathcal{N}_{+} \Psi\right\|\right)^{2},
\end{aligned}
$$

we then obtain

$$
\left|\left\langle e^{-i H_{N}^{\mathrm{aux}} s} \Phi^{\leq N},\left(H_{N}^{\mathrm{aux}}-H^{\mathrm{BF}}\right) e^{-i H^{\mathrm{BF}} s} \Phi\right\rangle\right| \leq C N^{-1 / 2}\left(\alpha^{\operatorname{aux}}(s) \alpha^{\mathrm{BF}}(s)\right)^{1 / 2}
$$

with some constant $C>0$. Applying Lemma 3.1 thus completes the proof.

\section{A Self-adjointness of the Hamiltonians}

Here we prove the relevant self-adjointness and domain properties of $H^{\text {aux }}$ and $H^{\mathrm{BF}}$, as well as a useful general Lemma.

Lemma A.1. Let $\left(M_{j k}\right)_{j, k \in \mathbb{N}} \in \ell^{2}(\mathbb{N} \times \mathbb{N})$. Then for any $\Phi \in D\left(\mathcal{N}_{+}\right)$

$$
\begin{aligned}
& \left\|\sum_{j, k \in \mathbb{N}} M_{j, k} a_{j} a_{k} \Phi\right\|_{\mathcal{F}_{+}} \leq\|M\|_{\ell^{2}}\left\|\mathcal{N}_{+} \Phi\right\|_{\mathcal{F}_{+}} \\
& \left\|\sum_{j, k \in \mathbb{N}} M_{j, k} a_{j}^{*} a_{k}^{*} \Phi\right\|_{\mathcal{F}_{+}} \leq\|M\|_{\ell^{2}}\left\|\left(\mathcal{N}_{+}+2\right) \Phi\right\|_{\mathcal{F}_{+}} .
\end{aligned}
$$


Proof. We only prove the first inequality, the second can be proved in a similar way. We have for any $\Psi \in \mathcal{F}_{+}$by the Cauchy-Schwarz inequality

$$
\left|\left\langle\Psi, \sum_{j, k \in \mathbb{N}} M_{j, k} a_{j} a_{k} \Phi\right\rangle\right| \leq\|M\|_{\ell^{2}}\left(\sum_{j, k \in \mathbb{N}}\left|\left\langle\Psi, a_{j} a_{k} \Phi\right\rangle\right|^{2}\right)^{1 / 2} .
$$

With (41) we get the claim.

Lemma A.2. Assume that $V, W \in L^{2}\left(T^{d}\right)$ are infinitesimally bounded relative to $-\Delta$. The operator $H_{N}^{\text {aux }}$ defined by the expression (26) is self-adjoint on $U_{N} D\left(H_{N}\right)$ and essentially selfadjoint on $D\left(H_{0}\right)$.

Proof. For the second claim, we prove that $H_{N}^{\text {aux }}$ is a perturbation of $-\frac{1}{2 m} \Delta_{x}+d \Gamma(-\Delta) \leq N$, the projection of $H_{0}$ to $\mathcal{H}_{+}^{\leq N}$, by a bounded operator.

For the quadratic terms in (26), this follows from the fact that $H_{N}^{\text {aux }}$ acts non-trivially only on $\mathcal{H}_{+}^{\leq N}$ and Lemma A.1 together with Parseval's identity, which yields

$$
\begin{aligned}
\left\|V_{j k 00}\right\|_{\ell^{2}(\mathbb{N} \times \mathbb{N})}^{2} & =\left\|V_{00 j k}\right\|_{\ell^{2}(\mathbb{N} \times \mathbb{N})}^{2} \\
& \leq \sum_{j, k \in \mathbb{N}_{0}}\left|\int_{T^{d}} \int_{T^{d}} \bar{\varphi}_{j}(y) \bar{\varphi}_{k}\left(y^{\prime}\right) V\left(y-y^{\prime}\right) d y d y^{\prime}\right|^{2} \\
& =\left\|V\left(y-y^{\prime}\right)\right\|_{L^{2}\left(T^{d} \times T^{d}\right)}^{2}=\|V\|_{L^{2}\left(T^{d}\right)}^{2}, \\
\left\|V_{j 0 k 0}\right\|_{\ell^{2}(\mathbb{N} \times \mathbb{N})}^{2} & \leq \sum_{j, k \in \mathbb{N}_{0}}\left|\left\langle\varphi_{j} \otimes \varphi_{0}, V\left(y_{1}-y_{2}\right) \varphi_{0} \otimes \varphi_{k}\right\rangle\right|^{2}=\|V\|_{L^{2}\left(T^{d}\right)}^{2} .
\end{aligned}
$$

For the linear term in (26) this follows from the bound $\left\|a\left(W_{x}\right) \Psi\right\|_{\mathcal{H}_{+}} \leq\|W\|_{L^{2}}\left\|\sqrt{\mathcal{N}_{+}} \Psi\right\|_{\mathcal{H}_{+}}$ by the same reasoning. Hence, $H_{N}^{\text {aux }}$ is self-adjoint on the domain of $-\frac{1}{2 m} \Delta_{x}+d \Gamma(-\Delta) \leq N$ and essentially self-adjoint on $D\left(H_{0}\right)$ by the Kato-Rellich theorem.

To obtain the first claim it is now sufficient to prove that the difference of $H_{N}^{\text {aux }}$ and $U_{N} H_{N} U_{N}^{*}$ is bounded relative to $d \Gamma(-\Delta) \leq N$, with relative bound zero. This difference consists of cubic (45) and quartic (46) terms involving $V$, and a quadratic term (44) with $W$. The cubic terms are bounded by an argument analogous to (38). The relative bound for $N^{-1 / 2} d \Gamma\left(Q W_{x} Q\right)^{\leq N}$ (i.e. (44) ) is a consequence of the bound for $\Psi \in D(d \Gamma(-\Delta)), \Phi \in \mathcal{H}_{+}^{\leq N}$, and $\lambda \geq 0$ (cf. [29, Eqs.2.9, 2.10])

$$
\begin{aligned}
\left|\left\langle\Phi, N^{-1 / 2} d \Gamma\left(Q W_{x} Q\right)^{\leq N} \Psi\right\rangle\right| \leq N^{-1 / 2}\left\|\mathcal{N}_{+}^{1 / 2} \Phi\right\|_{\mathcal{H}_{+}^{\leq N}}\left\|d \Gamma\left(\left(Q W_{x} Q\right)^{2}\right)^{1 / 2} \Psi\right\|_{\mathcal{H}_{+}^{\leq N}} \\
\leq \varepsilon\|\Phi\|_{\mathcal{H}_{+}^{\leq N}}\left\|d \Gamma\left((\lambda-\Delta)^{2}\right)^{1 / 2} \Psi\right\|_{\mathcal{H}_{+}^{\leq N}} \\
\leq \varepsilon\|\Phi\|_{\mathcal{H}_{+}^{\leq N}}\|d \Gamma(\lambda-\Delta) \Psi\|_{\mathcal{H}_{+}^{\leq N}},
\end{aligned}
$$

with $\varepsilon=\left\|(\lambda-\Delta)^{-1} W_{x}\right\|_{L^{2} \rightarrow L^{2}}=\left\|W_{x}(\lambda-\Delta)^{-1}\right\|_{L^{2} \rightarrow L^{2}}$. Since $\varepsilon$ goes to zero as $\lambda \rightarrow \infty$, because $W$ is infinitesimally $-\Delta$-bounded, this gives an infinitesimal bound. The quartic

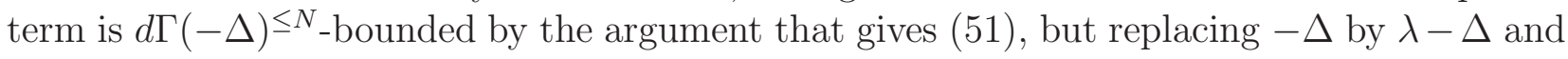
arguing as above. 
Proposition A.3. Let $V, W \in L^{2}\left(T^{d}\right)$, then $H^{\mathrm{BF}}-H_{0}$ is $\mathcal{N}_{+}$-bounded and $H^{\mathrm{BF}}$ is essentially self-adjoint on $D\left(H_{0}\right)$.

Proof. The first statement follows from Lemma A.1 as above. Consequently, there exists a constant $c$ such that $H^{\mathrm{BF}}+c \mathcal{N}_{+}$is self-adjoint and positive on $D\left(H_{0}\right)=D\left(H_{0}+c \mathcal{N}_{+}\right)$by the Kato-Rellich theorem, since $H^{\mathrm{BF}}-H_{0}$ is $H_{0}+c \mathcal{N}_{+}$-bounded with bound less than one.

Essential self-adjointness of $H^{\mathrm{BF}}$ can now be obtained by applying the commutator theorem [41, Thm.X.36], with $H^{\mathrm{BF}}+c \mathcal{N}_{+}$as a comparison operator. For this, it is sufficient to prove that

$$
\left|\left\langle\Phi,\left[\mathcal{N}_{+}, H^{\mathrm{BF}}\right] \Phi\right\rangle\right|=\left|\left\langle\Phi,\left[\mathcal{N}_{+}, H^{\mathrm{BF}}-H_{0}\right] \Phi\right\rangle\right| \leq C\left\langle\Phi, \mathcal{N}_{+} \Phi\right\rangle,
$$

for some constant $C>0$ and all $\Phi \in D\left(H_{0}^{1 / 2}\right)$. Since the commutator of $\mathcal{N}_{+}$with $H^{\text {Bog }}$ is again a quadratic operator, composed of the same terms up to signs, this follows from Lemma A.1 as above.

\section{References}

[1] N. Benedikter, M. Porta, and B. Schlein. Effective Evolution Equations from Quantum Dynamics. Springer Briefs in Mathematical Physics. Springer, 2016.

[2] C. Boccato, C. Brennecke, S. Cenatiempo, and B. Schlein. Complete Bose-Einstein condensation in the Gross-Pitaevskii regime. Commun. Math. Phys., 359(3):975-1026, 2018.

[3] C. Boccato, C. Brennecke, S. Cenatiempo, and B. Schlein. Bogoliubov theory in the Gross-Pitaevskii limit. Acta Math., 222(2):219-335, 2019.

[4] N. Bogoliubov. On the theory of superfluidity. J. Phys., 11(1):23, 1947.

[5] L. Boßmann, N. Pavlović, P. Pickl, and A. Soffer. Higher order corrections to the meanfield description of the dynamics of interacting bosons. J. Stat. Phys., 178(6):1362-1396, 2020 .

[6] L. Boßmann, P. Petrat, P. Pickl, and A. Soffer. Beyond Bogoliubov dynamics. arXiv:1912.11004, 2019.

[7] L. Boßmann, P. Petrat, and R. Seiringer. Asymptotic expansion of the low-energy excitation spectrum for weakly interacting bosons. arXiv:2020.09825, 2020.

[8] C. Brennecke, P.T. Nam, M. Napiórkowski, and B. Schlein. Fluctuations of N-particle quantum dynamics around the nonlinear Schrödinger equation. Ann. Inst. H. Poincaré C, Anal. Non Linéaire, 36(5):1201 - 1235, 2019.

[9] T. Chen and A. Soffer. Mean field dynamics of a quantum tracer particle interacting with a boson gas. J. Funct. Anal., 276(3):971-1006, 2019. 
[10] D.-A. Deckert, J. Fröhlich, P. Pickl, and A. Pizzo. Dynamics of sound waves in an interacting Bose gas. Adv. Math., 293:275-323, 2016.

[11] J. Dereziński and M. Napiórkowski. Excitation spectrum of interacting bosons in the mean-field infinite-volume limit. Ann. Henri Poincaré, 15(12):2409-2439, 2014.

[12] H. Fröhlich. Theory of electrical breakdown in ionic crystals. Proc. R. Soc. Lond. A, 160(901):230-241, 1937.

[13] J. Fröhlich, Z. Gang, and A. Soffer. Some Hamiltonian models of friction. J. Math. Phys., 52:083508, 2011.

[14] J. Ginibre and G. Velo. The classical field limit of scattering theory for non-relativistic many-boson systems. I. Commun. Math. Phys., 66(1):37-76, 1979.

[15] J. Ginibre and G. Velo. The classical field limit of scattering theory for non-relativistic many-boson systems. II. Commun. Math. Phys., 68(1):45-68, 1979.

[16] P. Grech and R. Seiringer. The excitation spectrum for weakly interacting bosons in a trap. Commun. Math. Phys., 322(2):559-591, 2013.

[17] M. Grillakis and M. Machedon. Pair excitations and the mean field approximation of interacting bosons, I. Commun. Math. Phys., 324(2):601-636, 2013.

[18] M. Grillakis and M. Machedon. Pair excitations and the mean field approximation of interacting bosons, II. Commun. PDE, 42(1):24-67, 2017.

[19] M. Grillakis, M. Machedon, and D. Margetis. Second-order corrections to mean field evolution of weakly interacting bosons, I. Commun. Math. Phys., 294(1):273, 2010.

[20] M. Grillakis, M. Machedon, and D. Margetis. Second-order corrections to mean field evolution of weakly interacting bosons, II. Adv. Math., 228(3):1788-1815, 2011.

[21] F. Grusdt and E. Demler. New theoretical approaches to Bose polarons. In M. Inguscio, W. Ketterle, S. Stringari, and G. Roati, editors, Proceedings of the international school of physics "Enrico Fermi", pages 325-411. Società Italiana di Fisica, 2016.

[22] K. Hepp. The classical limit for quantum mechanical correlation functions. Commun. Math. Phys., 35(4):265-277, 1974.

[23] E. Kuz. Exact evolution versus mean field with second-order correction for bosons interacting via short-range two-body potential. Differ. Integral Equ., 30(7/8):587-630, 2017.

[24] J. Lampart. The renormalised Bogoliubov-Fröhlich Hamiltonian. J. Math. Phys., 61(10):101902, 2020.

[25] M. Lewin. Mean-field limit of Bose systems: rigorous results. arXiv:1510.04407, 2015. 
[26] M. Lewin, P. T. Nam, and B. Schlein. Fluctuations around Hartree states in the mean field regime. Amer. J. Math., 137(6):1613-1650, 2015.

[27] M. Lewin, P. T. Nam, S. Serfaty, and J. P. Solovej. Bogoliubov spectrum of interacting Bose gases. Commun. Pure Appl. Math., 68(3):413-471, 2015.

[28] D. Mitrouskas, S. Petrat, and P. Pickl. Bogoliubov corrections and trace norm convergence for the Hartree dynamics. Rev. Math. Phys., 31(8), 2019.

[29] J. S. Møller. The translation invariant massive Nelson model: I. the bottom of the spectrum. Ann. H. Poincaré, 6(6):1091-1135, 2005.

[30] K. Mysliwy and R. Seiringer. Microscopic derivation of the Fröhlich Hamiltonian for the Bose polaron in the mean-field limit. arXiv preprint arXiv:2003.12371, 2020.

[31] P. T. Nam. Bogoliubov theory and bosonic atoms. arXiv:1109.2875, 2011.

[32] P. T. Nam and M. Napiórkowski. Bogoliubov correction to the mean-field dynamics of interacting bosons. Adv. Theor. Math. Phys., 21(3):683-738, 2017.

[33] P. T. Nam and M. Napiórkowski. A note on the validity of Bogoliubov correction to mean-field dynamics. J. Math. Pures Appl., 108(5):662-688, 2017.

[34] P. T. Nam and M. Napiórkowski. Norm approximation for many-body quantum dynamics: focusing case in low dimensions. Adv. Math., 350:547-587, 2019.

[35] E. Nelson. Interaction of nonrelativistic particles with a quantized scalar field. J. Math. Phys., 5(9):1190-1197, 1964.

[36] T. Paul and M. Pulvirenti. Asymptotic expansion of the mean-field approximation. Discrete Contin. Dyn. Syst. A, 39(4):1891-1921, 2019.

[37] S. Petrat, P. Pickl, and A. Soffer. Derivation of the Bogoliubov time evolution for a large volume mean-field limit. Ann. Henri Poincaré, 21(2):461-498, 2020.

[38] A. Pizzo. Bose particles in a box I. A convergent expansion of the ground state of a three-modes Bogoliubov Hamiltonian. arXiv:1511.07022, 2015.

[39] A. Pizzo. Bose particles in a box II. A convergent expansion of the ground state of the Bogoliubov Hamiltonian in the mean field limiting regime. arXiv:1511.07025, 2015.

[40] A. Pizzo. Bose particles in a box III. A convergent expansion of the ground state of the Hamiltonian in the mean field limiting regime. arXiv:1511.07026, 2015.

[41] M. Reed and B. Simon. Methods of modern mathematical physics:II Fourier analysis, self-adjointness. Academic Press, 1975.

[42] R. Seiringer. The excitation spectrum for weakly interacting bosons. Commun. Math. Phys., 306(2):565-578, 2011. 
[43] R. Seiringer. Bose gases, Bose-Einstein condensation, and the Bogoliubov approximation. J. Math. Phys., 55(7):075209, 2014.

[44] H. Spohn. Kinetic equations from Hamiltonian dynamics: Markovian limits. Rev. Modern Phys., 52(3):569-615, 1980.

[45] C. Zipkes, S. Palzer, C. Sias, and M. Köhl. A trapped single ion inside a Bose-Einstein condensate. Nature, 464(7287):388-391, 2010. 